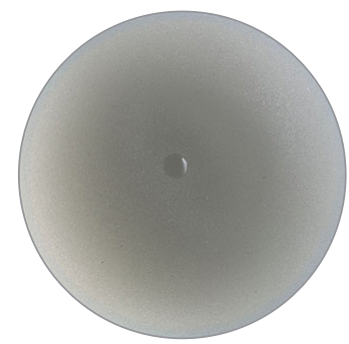

Palabras clave (DeCS)

Implantes de mama

Ultrasonografía mamaria

Hallazgos incidentales

\section{Key words (MeSH)}

Breast implants

Ultrasonography

mammary

Incidental finding
'Residente de Radiología, UPB/Cedimed. Medellín, Colombia.

${ }^{2}$ Radióloga Cedimed Medellín, Colombia.

\title{
Hallazgos ecográficos normales y complicaciones de implantes mamarios en Colombia
}

\author{
Normal Ultrasound Findings and Complications of Breast Implants in \\ Colombia
}

\author{
Valentina Múnera Orozco \\ Ximena Saldarriaga Jaramillo² \\ Catalina Mejía Soto
}

\section{Resumen}

La mamoplastia de aumento con implantes y la reconstrucción mamaria posmastectomía son algunos de los procedimientos quirúrgicos más frecuentes en cirugía plástica. Los implantes mamarios se encuentran entre los dispositivos médicos con mayor cantidad de informes por eventos adversos en el país. La extracción del implante es el estándar de oro ante la sospecha de la ruptura del mismo; sin embargo, hay poca claridad respecto a qué estrategias e imágenes son las adecuadas para la evaluación no invasiva de estos. La ecografía de alta resolución ha mostrado ser una alternativa para la evaluación inicial. Debido a la variabilidad de los implantes es necesario reconocer sus características imagenológicas. En este artículo se presentan los hallazgos ecográficos de los implantes, incluyendo aquellos que se pueden generar por marquillas o distintivos propios de la marca, y los propios de las complicaciones más comunes asociadas a ellos.

\section{Summary}

Implant augmentation mammoplasty and post-mastectomy breast reconstruction are some of the most frequent surgical procedures in plastic surgery. Breast implants are among the medical devices with the highest amount of reports of adverse events in our country. In suspicion of rupture, removal is the gold standard. However, there is little clarity regarding which strategies and images are adequate for non-invasive evaluation. High resolution ultrasound has shown to be an alternative for the initial evaluation. Due to the variability of the implants it is necessary to recognize their imaging characteristics. This article presents the findings of the ultrasound of the implants, including those that can be generated by tags or marks distinctive of the brand and those of the most common complications associated with them.

La mamoplastia de aumento es uno de los procedimientos quirúrgicos más frecuentes en cirugía plástica, tanto para cirugías de reconstrucción como para mamoplastia de aumento (1). Aunque no hay una cifra clara de cuántos pacientes se han realizado el procedimiento, Collett, en el JPRS2, estima que 35 millones de mujeres tenían implantes mamarios para el año 2016 (2).

Se han empleado varios criterios para clasificar los implantes mamarios utilizados en la mamoplastia: según su composición — silicona o solución salina-; según su envoltura - lisa o texturizada —; de acuerdo con su forma - anatómica o redonda - ; por su volumen — variable o fijo-; por el número de compartimentos - un solo lumen o bilumen - y, adicionalmente, cada marca tiene algunas características físicas que diferencian los implantes entre ellos (3), incluyendo válvulas para llenado o el sello de cierre en la parte posterior del implante
(4). Los implantes mamarios más comunes son los de un solo lumen, que contienen silicona como relleno (5).

En Colombia, de acuerdo con la base de datos del Programa Nacional de Tecnovigilancia 2005-2018 del Instituto Nacional de Vigilancia de Medicamentos y Alimentos (Invima), los implantes mamarios ocupan el sexto puesto entre los dispositivos médicos con mayor cantidad de reportes por eventos e incidentes adversos (6). La extracción del implante es el estándar de oro ante la sospecha de ruptura del mismo; sin embargo, hay poca claridad respecto a qué estrategias e imágenes son las adecuadas para la evaluación no invasiva de estos (7).

La resonancia magnética (RM) se ha considerado el estándar de oro no invasivo para la evaluación de los implantes mamarios de gel de silicona, con una sensibilidad mayor del $90 \%$ (8). Sus limitaciones son el costo, la claustrofobia y elementos metálicos no compatibles $(7,9)$. 
La mamografía no se considera la herramienta ideal para la evaluación de los implantes, por su baja sensibilidad en la detección de ruptura intracapsular del implante $(23 \%)(7,8,10)$. La ecografía de alta resolución, en manos de un radiólogo entrenado, es una alternativa más disponible, rápida, no dolorosa, menos costosa para la valoración y el seguimiento de implantes mamarios. Este procedimiento no expone al paciente a radiación ionizante y es mejor aceptada que la RM. Su sensibilidad está entre 59-70\%(9-11). Esta se limita por los transductores de alta frecuencia (12-18 MHz) que generan una mejor calidad de imagen, pero de resolución limitada a planos más superficiales, por lo que no evalúa todo el espesor del implante (7).

Por lo anterior, autores como Cilotti y colaboradores son claros en decir que la ecografía es la primera imagen que se debe realizar para la evaluación del implante, por un operador con experiencia, y la RM se debe usar solo cuando el diagnóstico sea incierto y la sospecha clínica de ruptura intracapsular sea muy alta (10) o cuando el médico tratante esté en desacuerdo clínico con el hallazgo ecográfico.

La evaluación por ecografía de los implantes mamarios incluye la revisión de su morfología, contornos, tejido mamario periimplante y axila $(5,10)$; la regularidad de los márgenes del implante, la cápsula, su contenido y homogeneidad del lumen, la presencia o no de líquido periprotésico, de silicona libre o granulomas en la mama o ganglios axilares $(5,10)$.
Este artículo tiene como objetivo revisar y describir los hallazgos ecográficos de los implantes mamarios, e incluye los registrados en el INVIMA, los que han salido del mercado, pero que fueron de amplio uso en la década 2010-2020, las marcas que han cambiado de nombre y los productos que no tiene registro INVIMA. Hasta la realización de este artículo, en 2020, en Colombia hay cinco compañías distribuidoras de implantes mamarios con registro INVIMA, y en total se comercializan ocho tipos de implantes —en Colombia, las marcas más usadas son Mentor, (comercializadora Johnson y Johnson), Natrelle, CUI, BRST (comercializadora Allergan), Motiva (Establishment Labs S. A.) y las comercializadoras Refimax y Hansbiomed Corp.- (6).

\section{Resultados}

Se presentan agrupados, según hallazgos físicos característicos (no por marca o empresa comercializadora), y se incluyen los que se pueden generar por marquillas o distintivos de la marca comercial, y los propios de las complicaciones más comunes asociadas a los implantes mamarios, cada uno con su imagen ecográfica correspondiente. Esta presentación tiene como propósito servir de guía para cuando el médico radiólogo se enfrente a una imagen de difícil diagnóstico (figuras 1-6).

\subsection{Generados por marquillas o distintivos de la marca comercial}

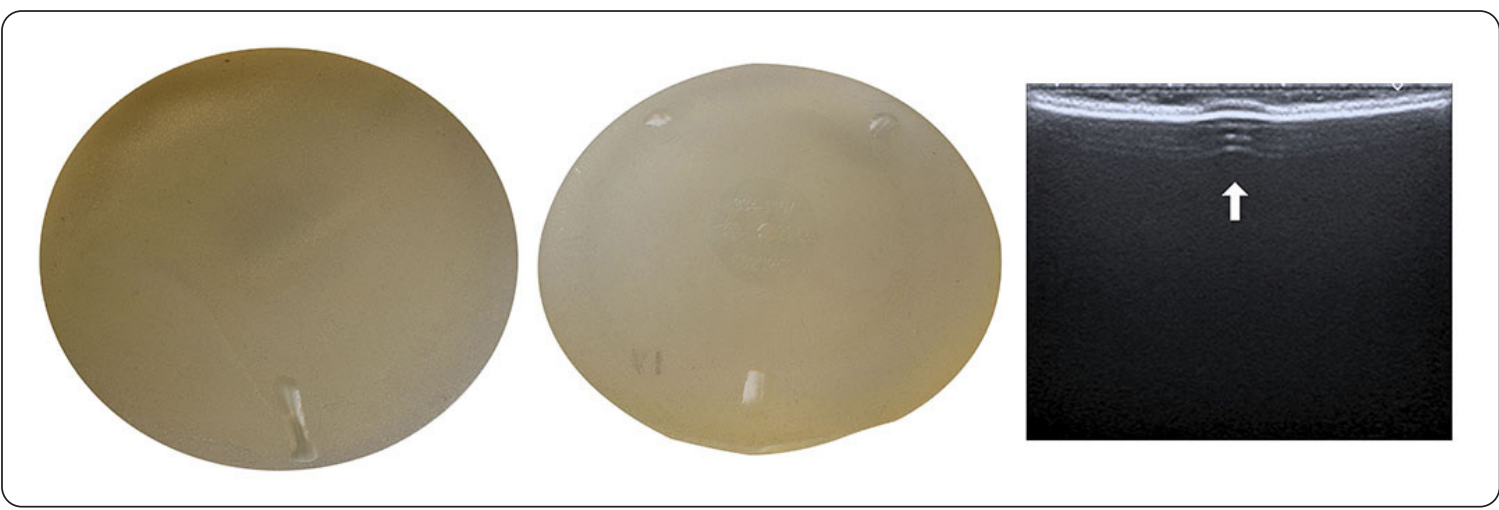

Figura 1. Prótesis anatómica marca Mentor. En la imagen de la derecha se ve el distintivo en el polo inferior cara anterior, vertical y lineal. En la imagen de la izquierda el sello posterior en el centro y dos distintivos redondos en el polo superior y otro vertical en el polo inferior. Ecográficamente se observan como una pequeña curva con una doble línea reflectiva. Sirve como guía intraoperatoria para evitar rotación.

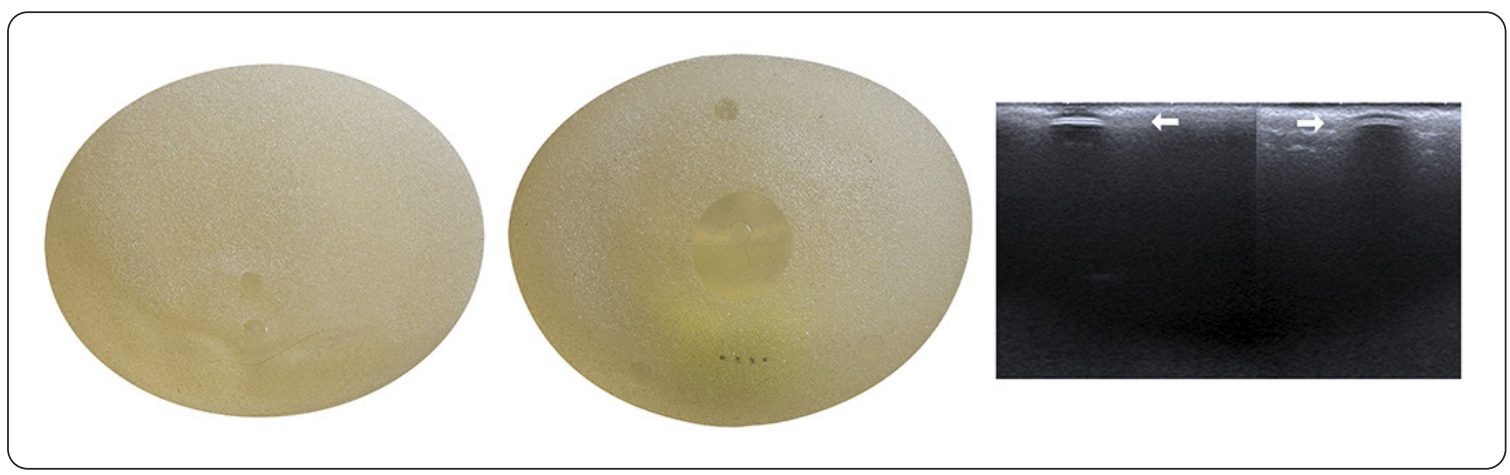

Figura 2. Prótesis anatómica, marca McGhan. En la imagen de la derecha se ven dos distintivos redondos en el polo inferior y cara anterior que se representa ecográficamente en la imagen superior como dos pequeñas curvas de cerca de 5 mm con una doble línea reflectiva. En la imagen de la izquierda, el sello posterior como un engrosamiento de la cápsula o una sola línea subcapsular ecogénica horizontal que se representa ecográficamente en la imagen inferior. 

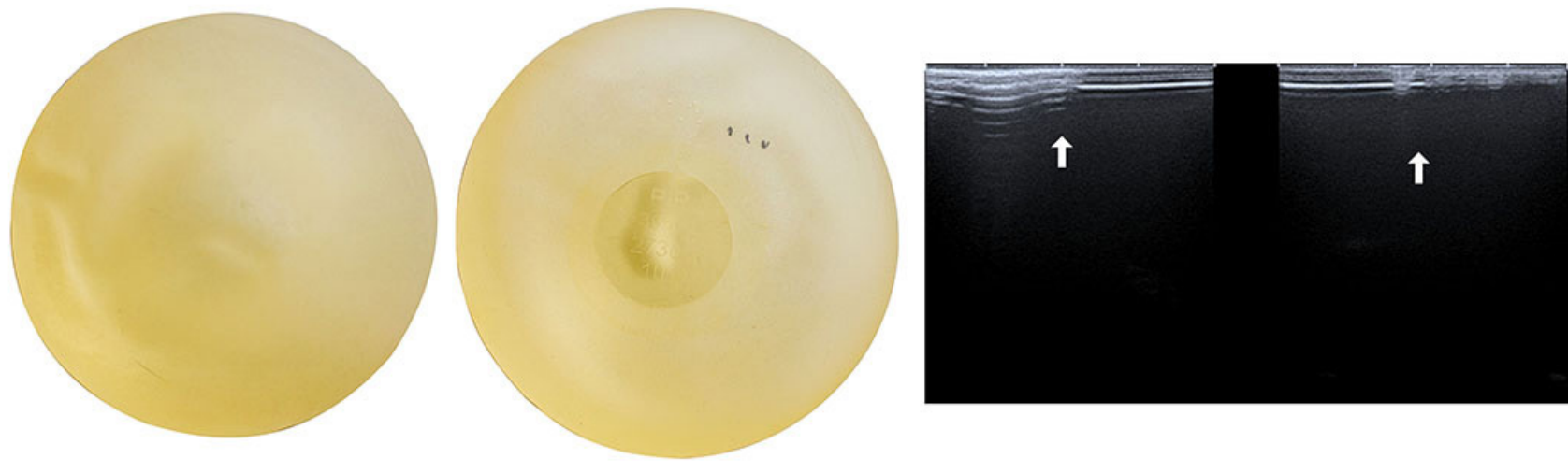

Figura 3. Prótesis redonda, marca PIP, no presenta distintivos en su cara anterior, pero sí el sello o marquilla posterior. Ecográficamente se presenta como una línea ecogénica paralela a la cápsula del implante, incompleta o un "engrosamiento posterior" de la cubierta del implante.

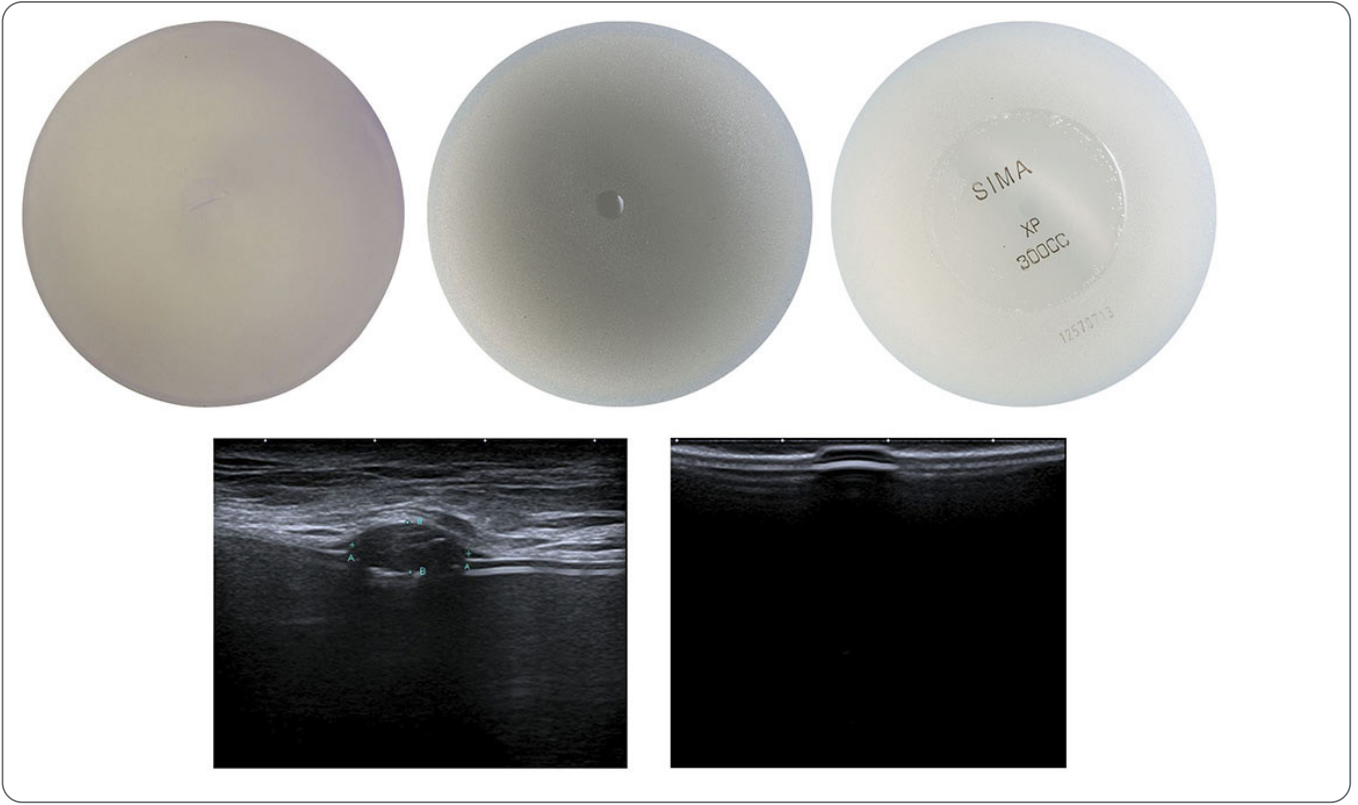

Figura 4. Prótesis redonda, marca SIMA, presenta dos formas diferentes de distintivos anteriores, redondos y pequeños. Ecográficamente se observa en la imagen de la izquierda ensanchamiento de la doble línea reflectiva con un centro anecoico pseudoquístico y en la imagen de la derecha como una pequeña curva de cerca de $5 \mathrm{~mm}$ con una doble línea reflectiva.

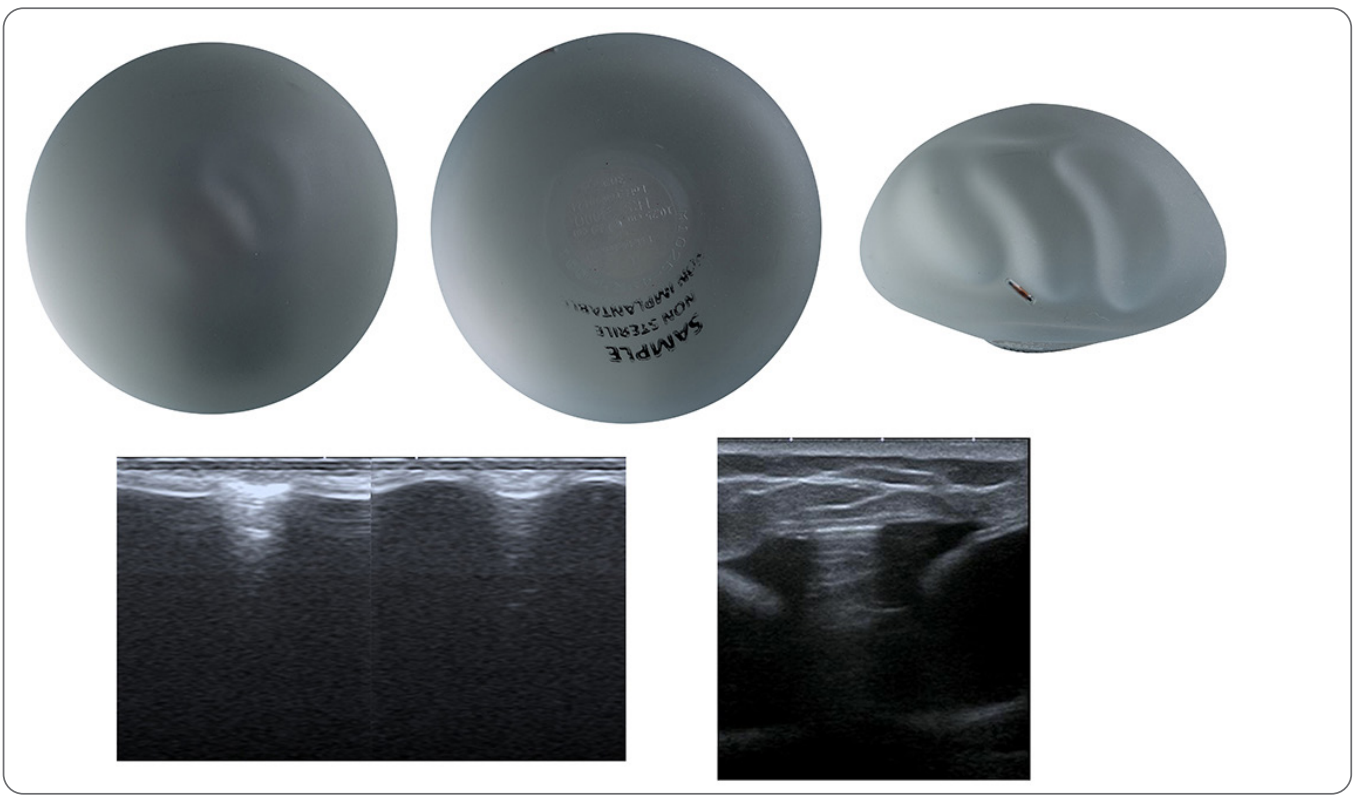

Figura 5. Prótesis redonda, marca Motiva, tiene un chip en cara lateral. Ecográficamente se identifica como imagen hiperecogénica que genera reverberación compatible con el chip (la imagen inferior derecha corresponde a un paciente con dicho implante). 


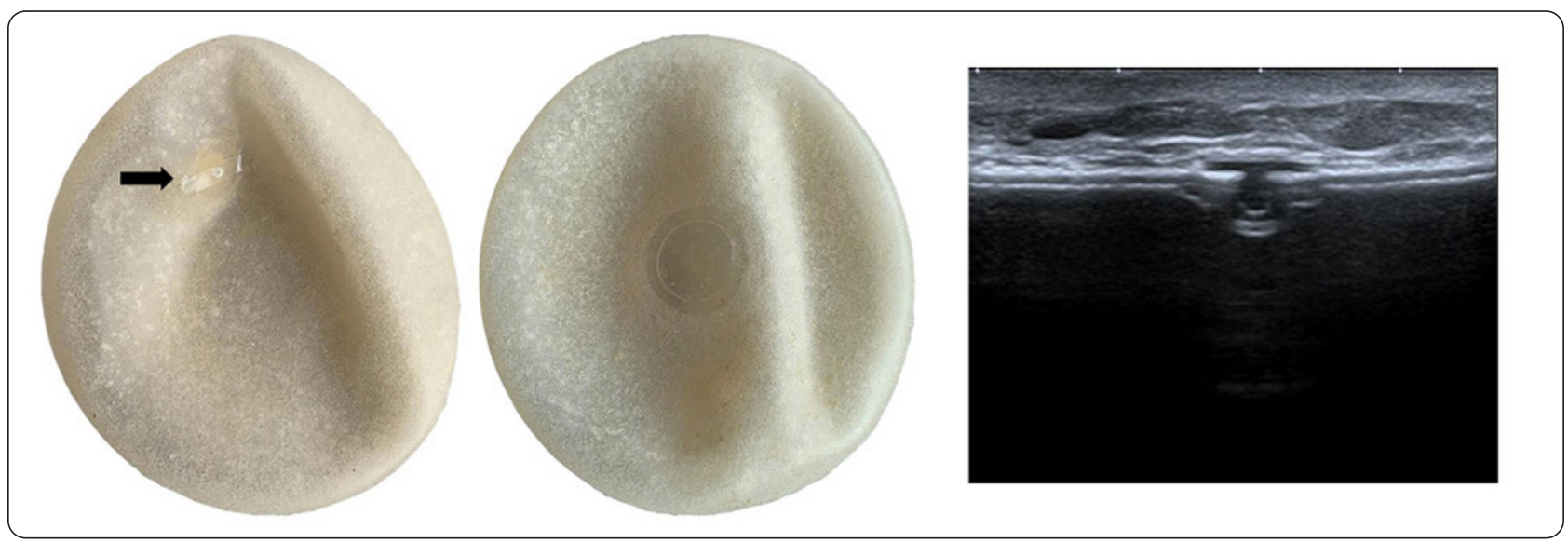

Figura 6. Prótesis de solución salina. Ecográficamente se demuestra una línea anecoica anterior a la cápsula del implante de una longitud de $5 \mathrm{~mm}$ y una pequeña curva invertida de cerca de $6 \mathrm{~mm}$ con una doble línea reflectiva que representa la válvula del implante (flecha negra).

\subsection{Hallazgos ecográficos propios de los implantes mamarios}

1.2.1. Pliegues, lobulaciones o invaginaciones. La imagen es variable y se produce cuando la superficie del implante se arruga durante la postura en un bolsillo creado por el cirujano, ya sea de localización subglandular (prepectoral) o subpectoral (debajo del músculo pectoral mayor). Son líneas ondulantes ecogénicas que se conocen como pliegues radiados, sin engrosamiento capsular asociado, rigidez, líquido periprotésico, ni dolor referido por la paciente (5) (figura 7).

1.2.2. Reverberación. Son imágenes ecogénicas que ocupan de manera lineal y horizontal todo el campo superficial de la imagen ecográfica, por reverberación del sonido a través de la cápsula. Dependen del grosor y calcificación de la cápsula del implante (del elastómero de silicona), de la densidad de la silicona y la resolución del ecógrafo. No indican ruptura del implante y son un hallazgo normal $(5,9)$ (figura 8).
1.2.3. Líquido periprotésico. Puede estar en cantidad variable, es fisiológico. Debe ser un líquido anecoico como el agua, sin ecos internos ni septos. Se cree que es el resultado de una respuesta inflamatoria y no es indicativo de ruptura (12) (figura 1).

1.2.4. Sello de silicona en la parte posterior del implante o marcador de superficie. Los implantes tienen una cápsula hecha de un elastómero de silicona que puede variar de 1-2 mm. En su mayoría, cuando se fabrican, tienen un hueco de varios centímetros en su parte posterior que se cierra tardíamente una vez se llene la luz del implante. Este parche, propio de cada marca, está hecho de un elastómero de silicona más grueso que se adhiere con una pega especial de silicona. Otros implantes se llenan con una aguja a través del parche y el pequeño agujero se cierra también con pega de silicona. Normalmente este hallazgo no se ve por ecografía por ser pequeño y muy posterior que se distorsiona con el haz de ultrasonido atravesando el implante $(4,9)$ (figura 9).

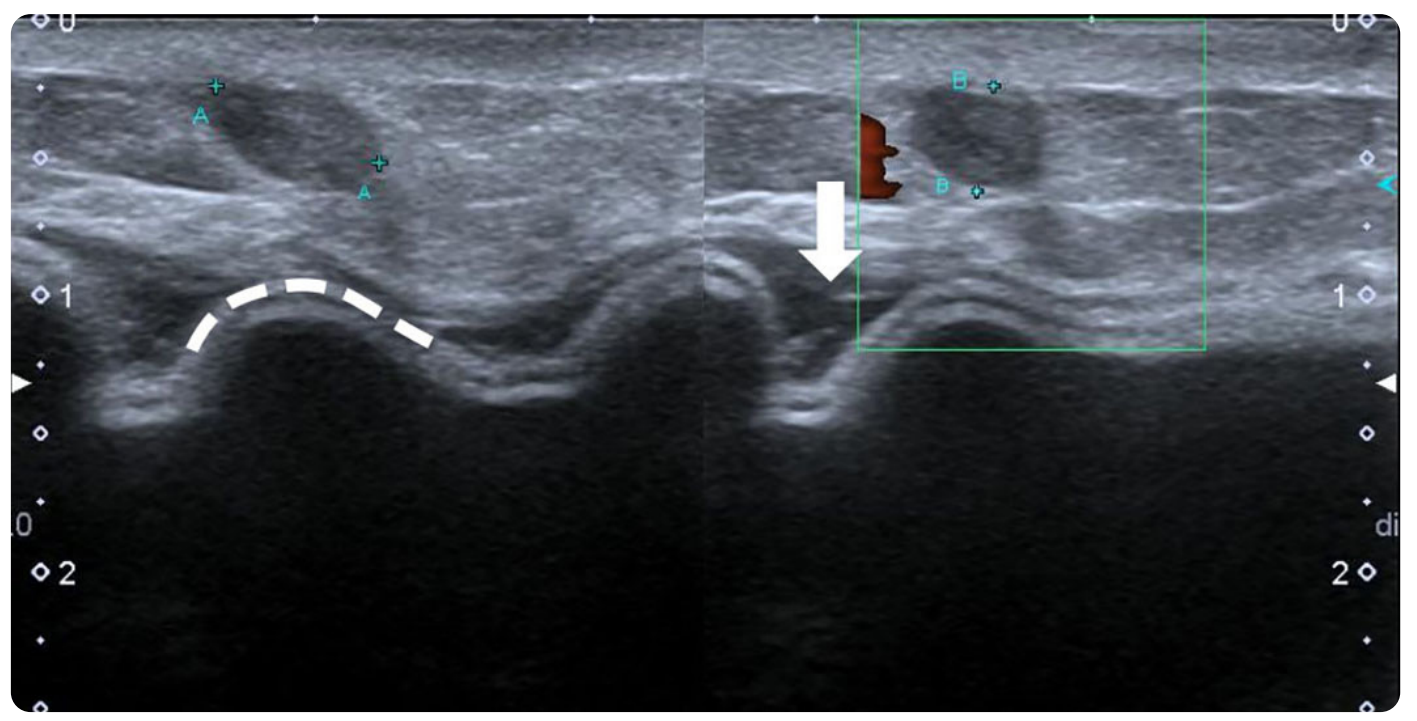

Figura 7. Se observa ecográficamente un implante con pliegues radiales prominentes (en línea discontinua blanca). En esta imagen se logra ver la cápsula fibrosa que se separa de la cápsula del implante por líquido periprotésico anecoico (flecha blanca). 


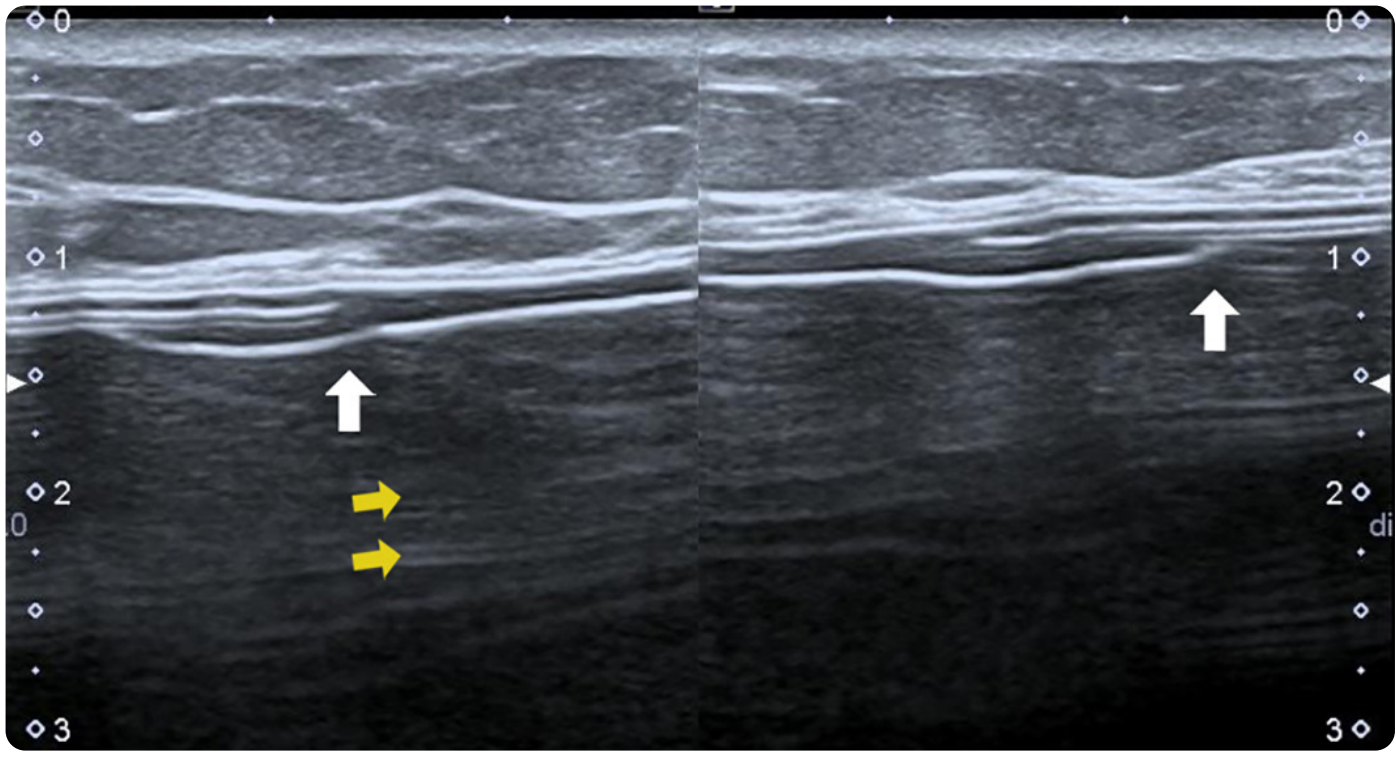

Figura 8. Imágenes ecogénicas que ocupan de manera lineal y horizontal todo el campo proximal y superficial de la imagen, por reverberación normal (flechas horizontales amarillas). Además, en la región anterior del implante se observa la marquilla posterior (flechas blancas) consistente con rotación del implante.

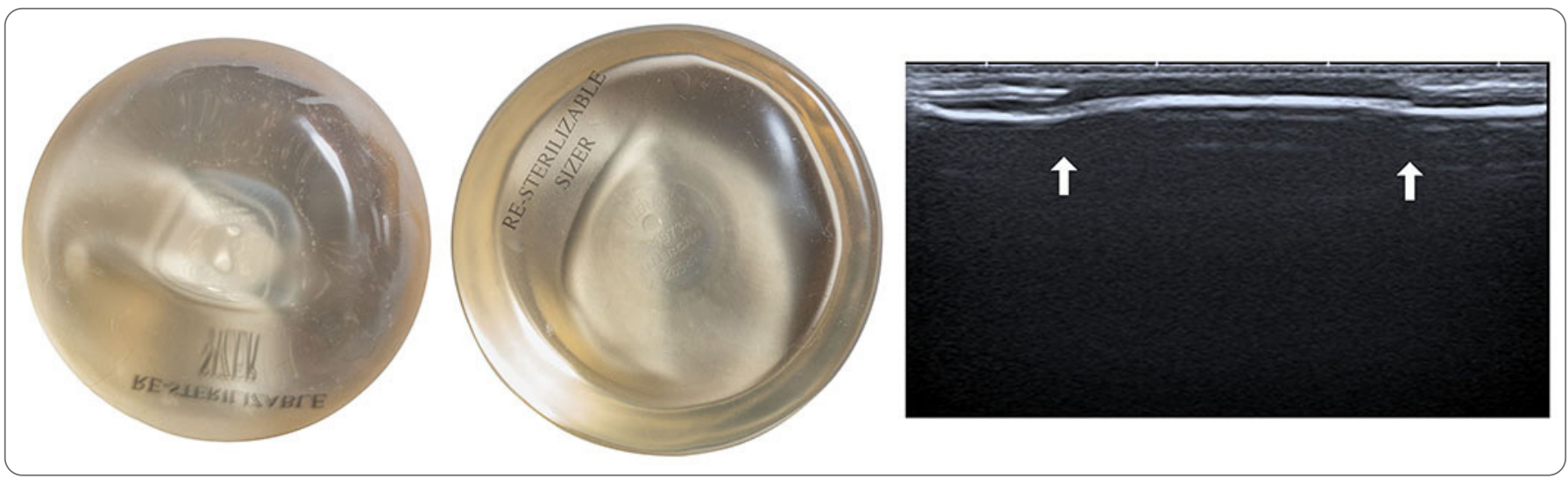

Figura 9. Implante Allergan. Ecográficamente hay rotación del implante en un eje de $180^{\circ}$. Se identifica la marquilla posterior como una línea ecogénica horizontal y paralela a la cápsula del implante, la cual está aparentemente discontinua. Parece un engrosamiento focal y posterior de la cápsula del implante.

\subsection{Hallazgos ecográficos asociados a complicaciones de los implantes mamarios}

1.3.1. Rotación. Es una de las complicaciones menos informadas y la tasa de rotación en la literatura es de 0-14\% (9). Los implantes de solución salina tienden a rotarse más frecuentemente (hasta $14 \%$ ) que los de gel cohesivo de silicona (2,6\%). Cuando se deshidratan, los implantes de solución salina se lobulan más, se encogen y se doblan, lo que facilita su rotación. Los implantes de gel cohesivo, por ser más rígidos, mantienen su forma y rotan menos (13). Se han descrito técnicas quirúrgicas, como colocar el polo superior o la mitad superior del implante debajo de la fascia superficial del músculo pectoral mayor en vez de subpectoral o retroglandular, para minimizar esta complicación (14).

Los marcadores en la superficie anterior del implante en el polo inferior están hechos para el monitoreo intraoperatorio por palpación, para asegurar una posición adecuada (14). Cuando un implante es redondo y se rota, por su forma más simétrica, no cambia mucho su apariencia clínica, pero un implante anatómico sí varía clínicamente al rotar y parece deformado $(11,13)$. La rotación puede ser sintomática o asintomática, con deformidad y asimetría, y puede requerir corrección quirúrgica (14). Una teoría sobre la causa de la rotación del implante es la falta del desarrollo de una capa de adhesión del tejido conjuntivo entre el implante y la cápsula, por lo tanto, permite que el implante se mueva libremente en el bolsillo. La textura de los implantes es variable y así también la formación de la capa de adhesión. Los implantes texturizados con poros más grandes disminuyen la posibilidad de rotación $(13,14)$. Los bolsillos quirúrgicos grandes, seromas o hematomas periprotésicos, masajes y ejercicio pueden favorecer la rotación (12).

El hallazgo ecográfico es una línea incompleta ecogénica subcapsular de una longitud variable de 1 a $4 \mathrm{~cm}$, y no debe confundirse con un desprendimiento capsular por una ruptura (figura 8). También puede observarse como una disrupción de la cápsula asociada a un óvalo o pequeños semicírculos que protruyen anteriormente (14) (figura 4).

1.3.2. Contractura capsular. La cápsula fibrosa es un tejido blando biológico que se produce después de la inserción del implante, 
como una respuesta fisiológica a un cuerpo extraño. La cápsula se puede engrosar o calcificar con el tiempo y se reconoce más fácilmente cuando hay líquido periprotésico $(10,13)$ (figura 7$)$.

El diagnóstico de contractura capsular es clínico y se utiliza la clasificación de Baker (10); sin embargo, el ultrasonido puede confirmar la sospecha clínica y proporcionar información adicional del implante. Ecográficamente se observa la contractura como una asimetría en la forma del implante con deformidad de sus contornos, lobulaciones o incremento en la profundidad de los pliegues y, además, de un engrosamiento capsular que puede variar, como una capa ecogénica gruesa que rodea la cubierta normal del implante $(5,7)$.

1.3.3. Deterioro de la sustancia interna del implante. Cilotti y colaboradores han observado heterogeneidad del contenido de silicona como focos puntiformes ecogénicos discontinuos intrasustanciales. No indican ruptura intracapsular (figura 10). Las pacientes con este hallazgo clínicamente están asintomáticas.

1.3.4. Gel bleed. Corresponde a una trasudación de moléculas pequeñas y microscópicas de silicona que atraviesan la cápsula intacta de silicona y la cápsula fibrosa, y migran a los ganglios axilares por vía linfática, en ausencia de ruptura del implante (5). Dan la apariencia de tormenta de nieve $(8,15)$ (figura 11).

Este hallazgo debe diferenciarse de una ruptura de un implante antiguo con migración de silicona a ganglios axilares. Las pacientes con este hallazgo generalmente son asintomáticas.

1.3.5. Ruptura. La ruptura del implante puede ser intracapsular (77-89\%) cuando se rompe la cubierta de silicona, pero queda confina- do dentro de la cápsula fibrosa del implante, o extracapsular (21\%) cuando el contenido atraviesa la cápsula fibrosa $(5,8,10)$. Las tasas de ruptura de los implantes aumentan con el tiempo transcurrido desde su inserción, pero pueden variar según los modelos y materiales $(7,8,16)$. Los implantes de gel cohesivo tienen menor posibilidad de ruptura, porque tienen una cubierta externa más gruesa y una capa de barrera además de un gel de silicona más viscoso (cohesivo) que los hacen estables en su forma y, en caso de romperse, el gel queda confinado dentro de la cápsula del implante (12). La tasa de ruptura en implantes anatómicos es entre 1-2,2 \% (12). La ruptura generalmente es asintomática.

En la ecografía se observa en una ruptura intracapsular una morfología irregular con pérdida de la continuidad del implante, con unas líneas ecogénicas ondulantes internas que representan partes de la cápsula flotando dentro de la silicona y silicona ecogénica móvil entre las dos capas del implante (10). Heterogeneidad de la sustancia interna con el signo "de la escalera", que son ecos lineales, múltiples $\mathrm{y}$ discontinuos en el lumen, sin filtración a ganglios axilares (10) (figura 12).

Ecográficamente en una ruptura extracapsular se observan signos de ruptura intracapsular y la asociación de masas intramamarias o axilares ecogénicas con pérdida del detalle posterior de la silicona o ruido posterior ecogénico, conocido como imagen de "tormenta de nieve", además de masas hipoecoicas asociadas a un ruido ecogénico alrededor de ellas $(10,17)$. El signo "de la tormenta de nieve" tiene una sensibilidad del $85,7 \%$ y una especificidad del $100 \%$ (14) y es posible que la apariencia de este signo esté relacionada con la cantidad de silicona en la masa o en el ganglio (15) (figura 11).

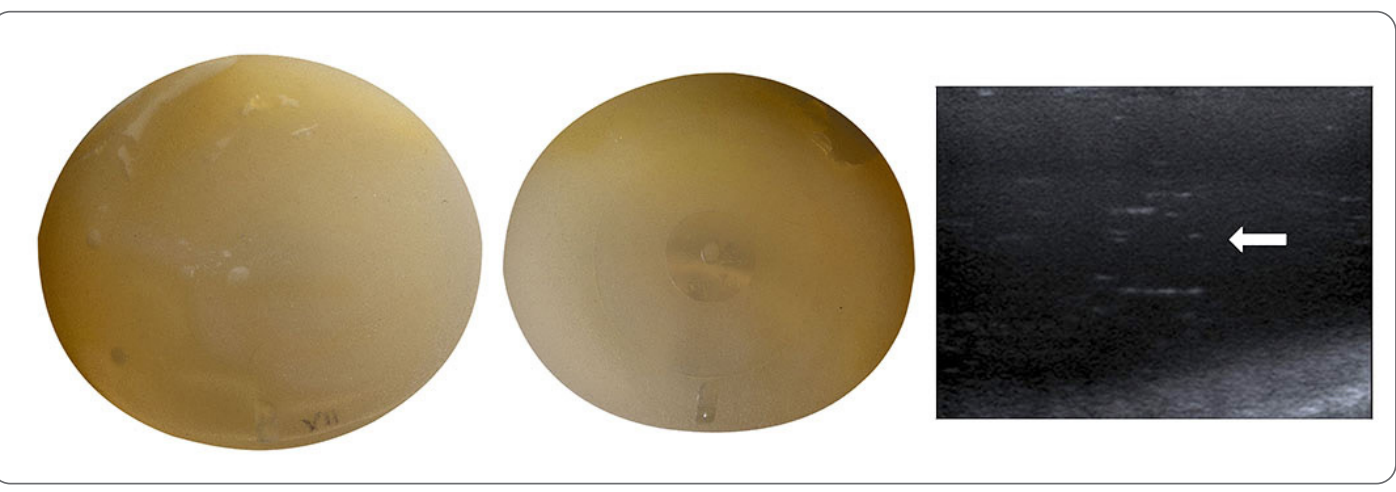

Figura 10. Macroscópicamente en los implantes se observan unas burbujas en su superficie. Ecográficamente se observan como heterogeneidad de contenido interno de silicona y focos puntiformes ecogénicos discontinuos intrasustanciales.

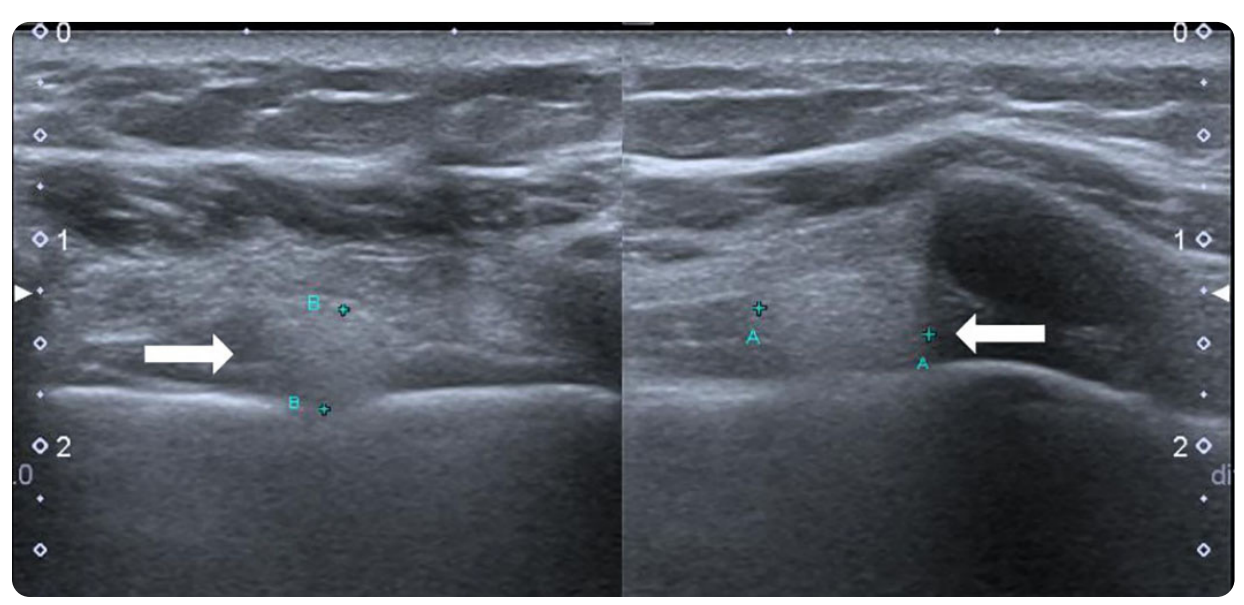

Figura 11. Ruptura extracapsular. Imagen ecográfica de siliconoma en la cadena mamaria interna después de ruptura de implantes. Imagen ecogénica con apariencia de tormenta de nieve y mala definición del borde posterior. Es la misma imagen para un siliconoma intratisular o en ganglios axilares. 

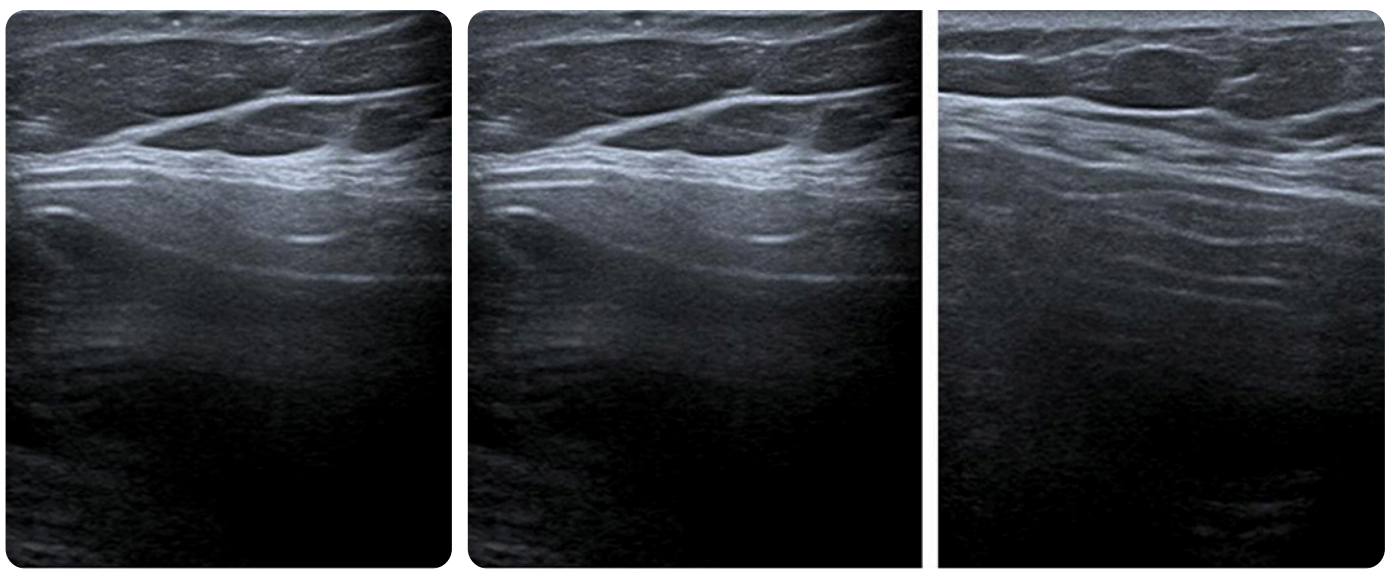

Figura 12. Ruptura intracapsular En la imagen de la izquierda pérdida de la integridad de implante con líneas paralelas ecogénicas ondulantes dentro de la luz del implante por ruptura capsular y silicona ecogénica contenida dentro de la capa externa del implante (flecha blanca). En la imagen de la derecha se observa el signo "de la escalera".

\section{Conclusión}

La ecografía de alta resolución es una técnica confiable y debería ser el primer método diagnóstico para valorar los hallazgos normales en las prótesis y sus complicaciones tempranas y tardías. Debe ser realizada por un operador experimentado.

Conocer la marca del implante en cada paciente y sus variaciones y características físicas y morfológicas ayudan a obtener un mejor diagnóstico ecográfico.

La RM se debe considerar en pacientes sintomáticas, con hallazgos ecográficos anormales y si hay dudas diagnósticas.

\section{Reconocimientos}

Las autoras agradecen al doctor Felipe Mesa Betancourt, cirujano plástico, por facilitarnos el material de soporte (implantes de mama) fotografiadas en el artículo, y al fotógrafo Andrés Cardona.

\section{Referencias}

1. Andrade E. Implantes mamarios de fijación. (Trabajo presentado en el Concurso de investigación, tecnología y ciencias básicas del XXXVI Congreso Nacional SCCP, Cartagena 2017). RCCP. 2018;24(2):4

2. Barbosa E. Nuevos horizontes de los implantes mamarios en la epoca del LACG. Página Resid. 2019;25(1):2.

3. Rocco N, Rispoli C, Moja L, Amato B, Iannone L, Testa S, et al. Different types of implants for reconstructive breast surgery. Cochrane Breast Cancer Group, editor. Cochrane Database Syst Rev [Internet]. 16 de mayo de 2016 [citado 5 de marzo de 2020]. Disponible en: http://doi.wiley.com/10.1002/14651858.CD010895.pub2

4. McNamara MP, Middleton MS. Ultrasound of breast implants and soft tissue silicone. Ultrasound Clin. 2011;6(3):345-68.

5. Shah A, Jankharia B. Imaging of common breast implants and implant-related complications: A pictorial essay. Indian J Radiol Imaging. 2016;26(2):216.

6. INVIMA. 966 Reportes de prótesis mamarias se han presentado en los últimos 10 años [Internet]. 2019. Disponible en: https://www.invima.gov.co/966-reportes-de-protesismamarias-se-han-presentado-en-los-ultimos-10-anos.

7. Bengtson BP, Eaves FF. High-resolution ultrasound in the detection of silicone gel breast implant shell failure: Background, in vitro studies, and early clinical results. Aesthet Surg J. 2012;32(2):157-74

8. Brown SL, Silverman BG, Berg WA. Rupture of silicone-gel breast implants: causes, sequelae, and diagnosis. Lancet Lond Engl. 1997;350(9090):1531-7.

9. Benito-Ruiz J, de Cabo F. Ultrasonography: A useful tool for plastic surgeons. Aesthetic Plast Surg [Internet]. 19 de marzo de 2014 [citado 2 de abril de 2020]. Disponible en: http://link.springer.com/10.1007/s00266-014-0300-z

10. Cilotti A, Marini C, Iacconi C, Mazzotta D, Moretti M, Giaconi C, et al. Ultrasonographic Appearance of Breast Implant Complications. Ann Plast Surg. 2006;56(3):243-7.
11. Sieber DA, Stark RY, Chase S, Schafer M, Adams WP. Clinical evaluation of shaped gel breast implant rotation using high-resolution ultrasound. Aesthet Surg J. 2017;37(3):290-

12. Juanpere S, Pérez E, Huc O, Motos N, Pont J, Pedraza S. Imaging of breast implants - a pictorial review. Insights Imaging. 2011;2(6):653-70.

13. Sampaio Góes JC. Breast implant stability in the subfascial plane and the new shaped silicone gel breast implants. Aesthetic Plast Surg. 2010;34(1):23-8

14. Hahn M, Kuner RP, Scheler P, Freidel K, Hoffmann G, Madjar H, et al. Sonographic criteria for the confirmation of implant rotation and the development of an implantcapsule-interaction ("interface") in anatomically formed textured breast implants with texturised Biocell-surface. Ultraschall Med Stuttg Ger 1980. 2008;29(4):399-404.

15. Klang E, Yosepovich A, Krosser A, Soffer S, Halshtok Neiman O, Shalmon A, et al Detection of pathologically proven silicone lymphadenopathy: ultrasonography versus magnetic resonance imaging: Imaging of silicone lymphadenopathy. J Ultrasound Med. 2018;37(4):969-75.

16. Caskey CI, Berg WA, Hamper UM, Sheth S, Chang BW, Anderson ND. Imaging spectrum of extracapsular silicone: Correlation of US, MR imaging, mammographic, and histopathologic findings. RadioGraphics. 1999;19(suppl 1):S39-51.

17. Dragoumis D, Assimaki A, Vrizas T, Tsiftsoglou A. Axillary silicone lymphadenopathy secondary to augmentation mammaplasty. Indian J Plast Surg. 2010;43(2):206.

\section{Correspondencia}

Valentina Múnera

Calle 31 \# 42A-61 Ciudadela San Diego

Medellín, Colombia

valentina.muneraorozco@gmail.com

Recibido para evaluación: 2 de julio de 2020

Aceptado para publicación: 15 de septiembre de 2020 